\title{
Analysis on Bot-A New Type of Community in the Chinese Internet Environment
}

\author{
Yilin Liu \\ Yunnan Arts University, the Film \& TV School, Kunming, Yunnan Province, China \\ 1020677154@qq.com
}

\begin{abstract}
The Chinese Internet differs from the Internet environment of other languages in that it has a communication behavior that is related to social bot but fundamentally different. It is a carefully categorized, original community where the manager sits behind an avatar and posts information to followers on a regular and quantitative basis with subjective tendencies. In this paper, a social platform in the Chinese Internet environment, the Bot on Sina Weibo, is used as an example as well as the street graffiti bot. Besides, this new type of community is analyzed through data analysis. At the same time, the reasons for the rise and fall of this new community were analyzed based on the two unique operational ideas. From the results, it was concluded that Street Graffiti Bot did not meet the operational expectations based on the two operational approaches. This suggests that if this new type of community is to be sustainable, other operational approaches and elements such as attracting traffic and interacting with Very Important Person are needed.
\end{abstract}

Keywords: Bot, Chinese Internet, Artificial Intelligence, Social Platform, Sina Weibo

\section{INTRODUCTION}

The Bot in the Chinese Internet environment is an original community of carefully categorised, managerially located avatars with a subjective tendency to post information regularly and quantitatively to fans.

Bot is an abbreviation for robot, and is in social platforms outside the Chinese Internet. Internet accounts called social bot by researchers originated as early AI bot accounts, referring to automated software agents[1] that act such as fostering fame[2]. On social platforms such as Twitter, the term bot often refers specifically to this type of highly influential phenomenon or account. In the early days, these bots sent social spam[3] on social media, and today's social bots have evolved in many new ways, often acting with a strong purpose such as opinion manipulation. Some researchers have argued that nearly a fifth of discussions on the Twitter platform in the 2016 US presidential election were bot-related[4]. Academic research on bots outside the Chinese environment has focused on this type of bot accounts, which are very different from bots in the Chinese Internet environment.

In the Chinese web environment, operators have redeveloped the "bot" form. These communities and accounts are often named "domain name + bot", and the operators are located behind a pre-planned avatar. The scripts are collected from fans or by operators themselves who then pretend to be bots to update them without the fans' knowledge. The content posted in the community is sometimes combined with current events and is subjective to the operator.

As a cultural phenomenon that emerged on Sina Weibo, bot accounts reached their peak of influence around 2020, with the emergence of many accounts with millions of followers that led the way on Sina Weibo for a period of time. Operators developed this form of community to a certain level and brought social value beyond information dissemination and the cultural sphere[5].

In terms of categories, bot-type accounts have gradually branched out from the initial celebrity blackmail category to developing types such as celebrity class, excerpt class and niche culture class. Some scholars also classify bots into six types: mutual aid, science popularization, sharing, emotional catharsis, marketing and reflecting social phenomena[6], regardless of how these bot class accounts are divided and categorized and certain commonalities among their operation methods. The following will analyze one of the bot accounts, Street Graffiti Bot, which the author 
personally runs, and conduct a qualitative and quantitative analysis of the community by collecting and organizing its background data. The operation of this community caters to the contradictory psychology that audiences are happy to receive regular and quantitative objective information and opinion-leadership-style opinion guidance, and operates with these two operational ideas.

The analysis of Bot on the Chinese Internet will bring some guidance on ideas for the operation of today's Internet accounts. The analysis on such a unique product of the Chinese Internet will also bring some thoughts to the development of various social platforms in western countries, and contribute to the comparative study of various social platforms

\section{STREET GRAFFITI BOT}

Before proceeding to the data analysis of the specific content, the author will analyze some macro data of Street Graffiti Bot to introduce the community from both the current situation and the audience. As the operator, the author has the backend data of Street Graffiti Bot. The time period of the data to be analyzed is limited to the period from March 1, 2020 to June 30, 2020, with the analysis on the operation of the community as a whole.

\subsection{Current status of operations}

Until June 30, 2020, Street Graffiti Bot has a total of 17400 followers, and 10900 of those followers were added during the period from March 1, 2020 to June 30, 2020 .

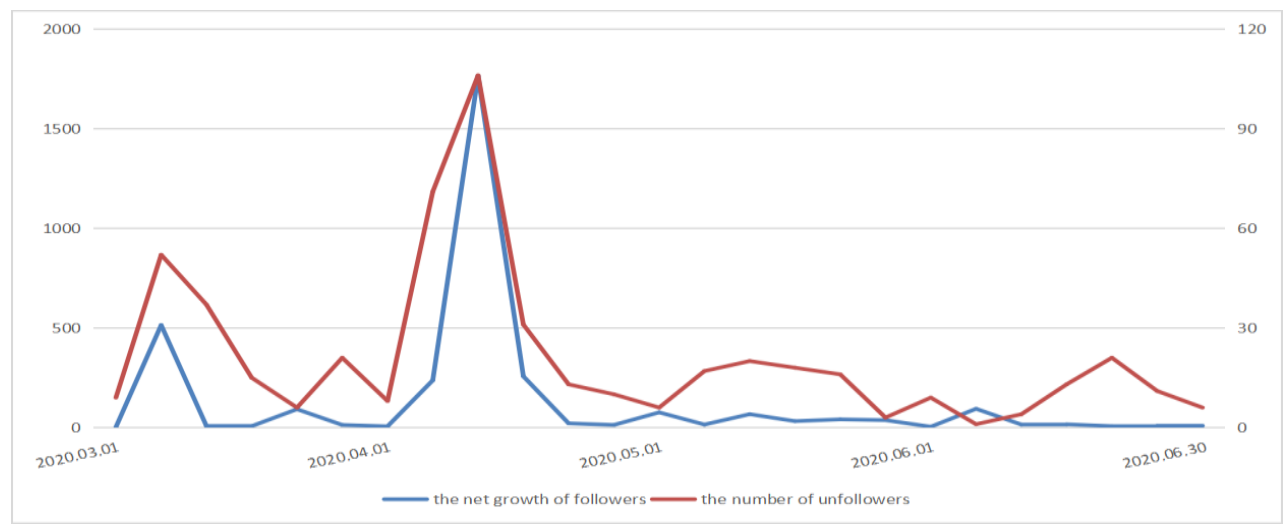

Figure 1 The net growth of followers and unfollowers from March 1 to June 30, 2020

The chart above shows the net growth of followers and unfollowers from the period of March 1, 2020 to June 30,2020 . We can see that there is a significant sudden fluctuation in the data in mid-April. Other than that, the total number of followers per day grew at the same rate. This is due to the fact that in mid-April, our account generated a breakout tweet. This tweet was retweeted by many internet celebrities with millions of followers. We will analyze this tweet specifically later.

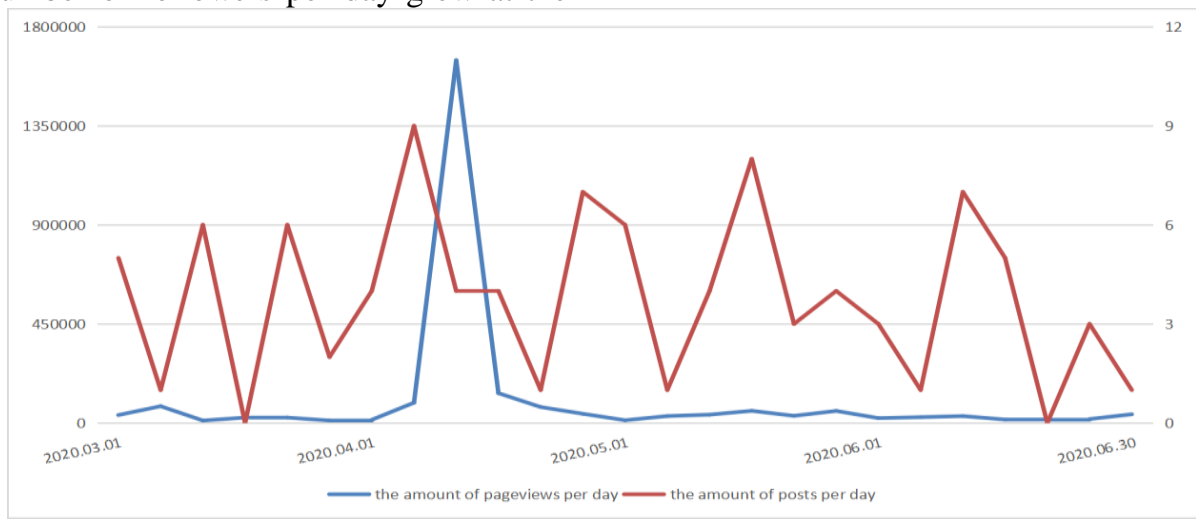

Figure 2 The amount of daily pageviews and posts on Sina Weibo from March 1 to June 30,2020

The chart above shows the amount of pageviews and posts per day from March 1 to June 30 of 2020. The comparison shows that the number of posts and pageviews are proportional to each other. However, when the number of posting decreases or even stops, the account will still maintain a certain amount of pageviews due to the Sina Weibo platform that attracts traffic.

During the selected time period, Street Graffiti Bot posted a total of 424 blogs and its number of pageviews 
reached 8.82 million in total without the operator updating at full speed. In this way, an average of less than four updates per day could get 70,000 pageviews on average each day. Besides, it's worth noting that this was done under the condition that Street Graffiti Bot had less than 10,000 followers on March 1, 2020, and in the following months the number of followers grew gradually.

Of course, this is related to the fact that the amount of pageviews from that aforementioned hot tweet pulled up the average. With Street Graffiti Bot reached 17,000 followers, a single tweet averaged around 12,000 pageviews.

\subsection{Audience profile}

A good fan base is a prerequisite and foundation for the continued healthy operation of an account, and in this respect Street Graffiti Bot has an advantage. The ratio of male to female fans of Street Graffiti Bot is roughly 40/60, which is related to the content that Street Graffiti Bot chooses to output. Without being intentional, the content we output attracts more female followers, which we hadn't predicted. Nevertheless, the ratio doesn't reach an imbalance, keeping the numbers relatively healthy.

With nearly $50 \%$ of fans aged $18-24$, the age of the fan base is relatively young. What is worrying is that this group may not have a high spending power. This in part makes it difficult for street graffiti bot to attract advertising attention, which is contrary to our ultimate goal of reaching profitability. At this stage, we can't make ends meet with our financial investment. The good news is that our fans have a huge potential spending power.

\section{OPERATIONAL IDEAS}

As mentioned earlier, the unique idea of operation is one of the greatest values of bot as a community. The operation of street graffiti bot caters to the conflicting mindset of an audience eager to receive timed, objective information and the subjective opinions of opinion leaders.

With this operational mindset, Street Graffiti Bot has gradually created sections and operations with operational characteristics and its own unique style since its launch in April 2019, without departing from the bot category. The community is run with the aim of growing followers, increasing readership and eventually posting thematically appropriate ads for profit. The channels thereafter are diversified into five forms, such as regular numbered updates, the online graffiti links that reflect abstraction, the "art is practical" links, the interactive links and lottery links.

\subsection{Catering to objectivity}

In order to cater to its fans' quest for objective information, Street Graffiti Bot has hatched three columns that are most characteristic of itself. Since most of the material for these columns comes from an objective world that is not under the control of the operator, who is more involved as a collector and collator, they have an extremely objective element.

Regular update sessions are a staple of Street Graffiti Bot. Based on the nature of the Internet community, the tweets sent rely on user submissions. The operator is responsible for screening and reviewing the content submitted by users based on certain criteria. After the review, the operator will post the images of the submissions in order, with the numbered text and @ contributor, which constitutes the regular update section. It ensures the amount of interaction between the operator's account and its followers and maintains the heat of the account.

The internet graffiti segment is almost the second most important section. While Street Graffiti Bot posts meaningful yet ridiculous street graffiti that people encounter in their daily lives, not all of it is written or painted on real walls. Many people "scribble" their emotions or whims on Sina Weibo, a social media platform, and Street Graffiti Bot sifts through them and retweets them with different numbers. It is more frequent than regular updates, preserving some of the fun or deeper thoughts and emotions.

The "art is practical" section is used to objectively document personal creations such as paintings, songs and photographs on the internet, with a greater emphasis on originality. The link was born from a submission of a picture on a wall with the words "Art is practical, but it's not necessary" spray painted in red. The operators wanted to use this link to encourage spontaneous acts of artistic creation.

\subsection{Catering to the subjective}

The subjective output is also what the operators of Street Graffiti Bot are looking for, and this subjective output is developed in two main sections.

The interactive session is when the operator will post a topic in the evening for the fans to express their respective views and express their different opinions. In addition, when it comes to the number of regular updates with a specific symbolic meaning, the operator will also occupy this number to post tweets, publish some of what they see and feel, and interact with fans. This link has more subjective ideas of the operator, which increases the amount of interaction and fan stickiness while outputting opinions. 
In the Sina Weibo platform, accounts are often operated with a lottery, and street graffiti bot often uses the Sina Weibo platform lottery tool at some special points in time to conduct a lottery. This can attract clicks and retweets from netizens and build up more buzz and discussion, thus spreading the word and increasing the number of fans. The copywriting of this link will also often carry the operator's strong subjective thoughts, outputting some ideas and opinions.

\section{CASE STUDIES}

Macro narratives often carry a certain amount of ambiguity, and in this section two specific cases will be analysed to show in detail how the community of street graffiti bot operates according to operational ideas.

\subsection{Catering to the desire for objectivity}

Street Graffiti Bot posted a regular series of updates "The wall at night" on April 5, 2020. In addition, it began being retweeted by dozens of internet celebrities with millions of fans on April 8, 2020. Besides,it brought us thousands of new followers within a week of its release, and the total number of retweets, replies and likes accumulated to a total of 10,000. It was one of Street Graffiti Bot's most successful regular series of updates to date, and the account's most successful tweet to date.

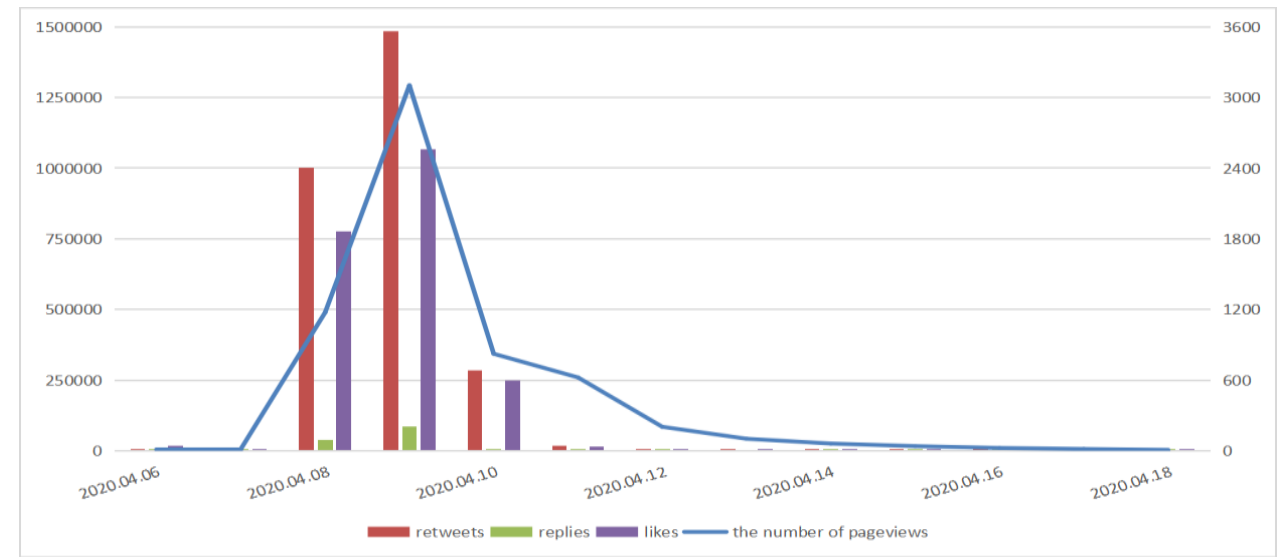

Figure 3 Reweets, replies, likes and pageviews of the post"The wall at night"

Here the author will sort out this kind of phenomenon. What can be summarized is that the decisive factor for this tweet to reach such a large number of readers is that dozens of internet celebrities retweeted and diverted it. This may be a shortcut that can boost the number of followers and attention. But unfortunately, this shortcut is almost impossible to replicate when the operator is not a team and is only a personal operator with very little capital investment. The only way to replicate this phenomenon again is to keep improving the quality of the content.

\subsection{Catering to the desire for subjective opinions}

A proper sweepstake can bring us more readers and new followers, express our attitude of continuous operation, and is a way to give back to our followers. Along with the sweepstakes, the operator can even make subjective thought output through copy editing and the meaning implied by the prize.

A raffle at the end of February 2020 deserves to be analyzed as a typical case study. In this event, Street Graffiti Bot drew three levels of prizes, five in total, and spent roughly 300 RMB. For example, Prizes such as spray paint cans, along with relevant scripts, hinted at some libertarian ideas. 


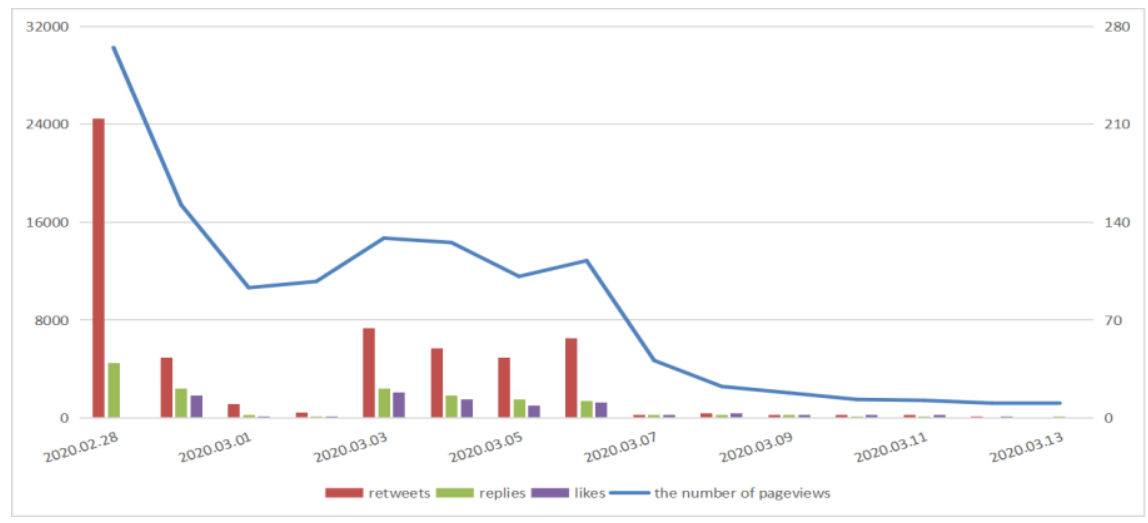

Figure 4 The post of raffle from February 28 to March 13, 2020

The total number of pageviews for this sweepstakes was at 137,900 , with 700 retweets, likes and comments in total. The pageviews and retweets peaked at the time the tweet was sent out. The numbers picked up again afterwards due to the secondary retweets that were made to build momentum. Such numbers may be passable, but they are still a long way from the desired effect. The number of retweets and likes could be called good with just under 10,000 followers at the time, but it's still a long way from excellence.

The most unsatisfying part for it is the growing number of followers which makes operators understand some reality of sweepstakes that it is impossible to try to get extremely viscous followers by simply sweepstakes. Besides, great view output can contribute to some data growth.

\section{DISCUSSION}

As a kind of original community with a detailed classification, the operator is located behind the virtual image and publishes information to fans at regular intervals with a subjective tendency. As a kind of pop culture on the spur of the moment, the content output of bots is mostly unsustainable, and they often rely on the contributions of the audience. Once fans lose their enthusiasm for contributing or there is less material to update, the operation of bot accounts will become problematic.

In today's age of information explosion, some audiences are certainly happy to receive regular and quantitative information updates and tweets devoid of subjective emotion. They are used to limiting the scale of information they access and watching more objective news. Because we are human, people are also paradoxically eager to reach consensus within our communities, and to see influential opinion leaders agree with and be influenced by the views of themselves. Based on this underlying logic, Bot caters well to these seemingly contradictory needs and follows through with operating in a way that caters to these mindsets. Street
Graffiti Bot has also achieved great results in terms of spreading its message and data.

However, it can be seen that when only such operational ideas are relied upon to grow a community, this is far from enough. They cannot keep the community hot in the long run. Street Graffiti Bot has proven this point in its later years of operation when it failed to achieve the expected growth in numbers. Perhaps when these ideas are borrowed, a mix of other operational methods such as traffic introduction and interaction with Very Important Person is more likely to lead to a longer term growth of an account or community.

\section{CONCLUSION}

In conclusion, the paper researched a social platform in the Chinese Internet environment through an example of the street graffiti bot. The results showed that Street Graffiti Bot did not meet the operational expectations based on the two operational approaches. This suggests that if this new type of community is to be sustainable, other operational approaches and elements such as attracting traffic and interacting with Very Important Person are needed.

Although the heat dies down and the internet continues to evolve, bot communities will probably die out. However, the author believes that the discussion on bot and the analysis of its operation ideas will continue with the development of artificial intelligence and the Internet. The research on the link between bot and AI may only be just beginning. This will also be the direction of the author's attention and research in the future.

\section{REFERENCES}

[1] Geiger R S. Bot-based collective blocklists in Twitter: the counterpublic moderation of harassment in a networked public space[J].Information, Communication \& Society, 2016, 19(6). 1. 
[2] Finger L. Do evil-the business of social media bots[J]. Forbes, 2015.

[3] Tynan D. Social spam is taking over the Internet[J]. IT World, Apr, 2012, 4.

[4] Bessi A, Ferrara E. Social bots distort the 2016 US Presidential election online discussion[J]. First monday, 2016, 21(11-7), 11.
[5] Wang Xingyi.Study on the social value of Weibo bot [J].New Media Research,2020,6(12):85-86, 2.

[6] Ren Qiyue.A study on the significance of self-disclosure in Weibo bot on subjective well-being[J].Digital World,2020(11):47-48, 1.

[7]https://weibo.com/p/1005056196204883/manage?fro $\mathrm{m}=$ page_100505\&mod=TAB\#place 